\title{
Micro-CT imaging of breast tumors in rodents using a liposomal, nanoparticle contrast agent
}

\author{
Ehsan Samei ${ }^{1,2,3,4}$ \\ Robert S Saunders' \\ Cristian T Badea ${ }^{2,5}$ \\ Ketan B Ghaghada ${ }^{2,5 \dagger}$ \\ Laurence W Hedlund ${ }^{2,5}$ \\ Yi Qi ${ }^{2,5}$ \\ Hong Yuan ${ }^{6}$ \\ Rex C Bentley ${ }^{7}$ \\ Srinivasan Mukundan $\mathrm{Jr}^{2,3,5 *}$ \\ 'Carl E Ravin Advanced Imaging \\ Laboratories, ${ }^{2}$ Department of \\ Radiology, ${ }^{3}$ Department of Biomedical \\ Engineering, ${ }^{4}$ Department of Physics, \\ ${ }^{5}$ Center for In Vivo Microscopy, \\ ${ }^{6}$ Department of Radiation Oncology, \\ ${ }^{7}$ Department of Pathology, Duke \\ University, Durham, NC, USA; \\ tCurrent address: School of Health \\ Information Sciences, University \\ of Texas Health Science Center at \\ Houston, Houston, TX, USA; *Current \\ address: Department of Radiology, \\ Brigham and Women's Hospital, \\ Boston, MA, USA
}

Correspondence: Ehsan Samei 2424 Erwin Road, Suite 302 (DUMC Box 273I), Durham, NC 27705

$\mathrm{Tel}+\mathrm{I} 9196847852$

Fax + I 919684 I492

Email samei@duke.edu
This article was published in the following Dove Press journal:

International Journal of Nanomedicine

18 November 2009

Number of times this article has been viewed

\begin{abstract}
A long circulating liposomal, nanoscale blood pool agent encapsulating traditional iodinated contrast agent ( $65 \mathrm{mg} \mathrm{I} / \mathrm{mL}$ ) was used for micro-computed tomography (CT) imaging of rats implanted with R3230AC mammary carcinoma. Three-dimensional vascular architecture of tumors was imaged at 100-micron isotropic resolution. The image data showed good qualitative correlation with pathologic findings. The approach holds promise for studying tumor angiogenesis and for evaluating anti-angiogenesis therapies.
\end{abstract}

Keywords: molecular imaging, liposome, angiogenesis, breast cancer, small animal imaging, micro-CT

\section{Introduction}

Angiogenesis plays an important role in breast tumor growth and in the process of metastasis. ${ }^{1}$ However, imaging angiogenesis using X-ray-based techniques has proved extremely difficult due to the small amount of contrast enhancement and the limited size of the tumor vessels. To surmount these difficulties, several conditions need to be met: a high concentration of contrast agent to provide adequate imaging signal, a stable contrast enhancement during the imaging procedure, and an imaging system with high spatial, contrast, and temporal resolution.

In the past few years, liposomes have been studied as excellent vehicles for drug delivery. ${ }^{2-7}$ They have also been used as contrast agents in computed tomography (CT) and magnetic resonance (MR) imaging, ${ }^{8-10}$ and as a delivery mechanism of contrast molecules to tumor regions. ${ }^{11}$ Some members of this group have previously developed and evaluated a long circulating liposomal-based, nanoparticle contrast agent for blood pool imaging. ${ }^{10}$ The agent has longer in vivo residence time than traditional contrast agents. The hypothesis of this study was that this new contrast agent would have utility in breast cancer imaging, providing long-lasting opacification of tumor microvasculature in micro-CT images of rats with implanted mammary tumors. The purpose of the study was to perform a proof-of-principle imaging experiment with this contrast agent by obtaining high-resolution images of mammary tumors in the rat model and qualitatively compare the images to histological specimens.

\section{Materials and methods}

\section{Contrast agent formation}

A lipid mixture (200 mM) consisting of 1,2-dipalmitoyl-sn-glycero-3-phosphocholine (DPPC), cholesterol, and 1,2-distearoyl-sn-glycero-3-phosphoethanolamine-N[methoxy(polyethylene glycol)-2000] (DSPE-MPEG2000) in the molar ratio 55:40:5 
was dissolved in ethanol at $70{ }^{\circ} \mathrm{C}$. The ethanol solution was then hydrated for two hours with Iopamidol solution (Isovue ${ }^{\circledR}$ 370, Bracco Diagnostics, Princeton, NJ, USA). Liposomes were extruded in a $10-\mathrm{mL}$ Lipex Thermoline extruder (Northern Lipids, Vancouver, British Columbia, Canada) with five passes and seven passes, respectively, through a 200- and a 100-nm Nuclepore membrane (Waterman, Newton, MA). Un-encapsulated iodine was then removed with a MicroKros ${ }^{\circledR}$ filtration module (Spectrum Labs, Rancho Dominguez, CA, USA) with a 400-kDa cut-off. Further detail on this synthesis process can be found in an earlier publication. ${ }^{10}$

The size distribution of the liposomes in the final formulation was determined by dynamic light scattering using a ZetaPlus Analyzer (Brookhaven Instruments, Inc., Chapel House, UK). The resultant liposomes were found to be $113 \mathrm{~nm}$ in size with a polydispersity index of 0.14 . The iodine concentration in the liposomal formulations was $65 \pm 5 \mathrm{mg} / \mathrm{ml}$, assessed based on the absorption of ultraviolet light at $242 \mathrm{~nm}$ measured with an ultraviolet-visible light spectrophotometer.

\section{In vivo studies}

All animal procedures were performed using protocols approved by the Duke University Institutional Animal Care and Use Committee. Eight female Fischer 344 rats (National Cancer Institute, Frederick Cancer Center, Frederick, MD, USA) with a weight of 126.6-145.5 g (140 g average) were used for the study. Each rat was implanted with the R3230AC Rat Mammary Adenocarcinoma. ${ }^{12}$ For tumor implantation, small pieces of tumor tissue (1-2 $\left.\mathrm{mm}^{3}\right)$ were obtained from tumor-bearing donor rats and transplanted subcutaneously in the right flank of each recipient animal. The animals were imaged approximately three weeks after implantation when the tumors reached $1-1.5 \mathrm{~cm}$ in diameter. They were imaged twice; at one and four hours after contrast injection.

\section{Animal procedures}

The rats were anesthetized using an intra-peritoneal $50 \mathrm{mg} / \mathrm{kg}$ injection of sodium pentobarbital and $2 \mathrm{mg} / \mathrm{kg}$ butorphanol. Following endotracheal intubation, anesthesia was maintained with $2 \%-3 \%$ isoflurane, delivered by a custom-made ventilator. ${ }^{13}$ Animals were mechanically ventilated at $90 \mathrm{breaths} / \mathrm{min}$ and at a tidal volume of $1.0 \mathrm{ml}$.

The iodinated liposomes were delivered via injection into a microcatheter inserted into the tail vein at a volume dose of approximately $0.02 \mathrm{ml} / \mathrm{kg}$ of animal weight. This was chosen as a standard volume for infusion as it results in adequate opacification of the blood vessels and tumors, and was well tolerated by the animals.

Animals were placed vertically in a cradle. Flexible tubes carrying anesthesia gas and wires conveying physiologic telemetric signals were suspended from above to allow free rotation of the animal throughout the $190^{\circ}$ rotation (Figure 1). All physiological telemetry data were displayed on a computer (LabVIEW, National Instruments, Austin, TX, USA). The monitored physiologic parameters were airway pressure tracing, measured with a solid-state pressure transducer on the breathing valve, and ECG, measured by electrodes taped to the footpads. Body temperature was maintained at $36.5^{\circ} \mathrm{C}$ with heat lamps, an oral thermistor, and feedback controller (Digi-Sense $^{\circledR}$, Cole-Parmer, Chicago, IL, USA).

Following the study, the animals were euthanized with an overdose of nembutal. Tumors were excised, sectioned into 5 to 10 micron slices, and stained using hematoxilin and eosin (H\&E) staining. Histological analysis was performed on the stained slices.

\section{Micro-CT system}

The micro-CT system used, described previously ${ }^{14}$ consisted of a high-flux, rotating anode X-ray tube (Philips SRO 09 50, Cleveland, OH, USA) with a dual 0.3/1.0-mm focal spot. The tube was capable of $10 \mathrm{msec}$ exposures to minimize cardiac motion blur. The tube was coupled to a cooled charge-coupled device (CCD) camera with a $\mathrm{Gd}_{2} \mathrm{O}_{2} \mathrm{~S}$ phosphor on a fiberoptic coupler with 3:1 de-magnification ratio (X-ray ImageStar, Photonics Science, East Sussex, UK). The camera had a $106 \mathrm{~mm}^{2}$ active field-of-view with a pixel size of $51 \mu \mathrm{m}$. The tube and detector were mounted in the horizontal plane on aluminum frame components (80/20 Inc., Columbia City, IN, USA) (see Figure 1).

The animal was centered vertically at $40 \mathrm{~mm}$ from the detector face. The distance between the animal and X-ray source was $480 \mathrm{~mm}$. This configuration essentially eliminated scatter, and focal spot blur by matching the penumbra to the Nyquist limiting frequency of the detector. ${ }^{14,15}$

\section{Image acquisition and reconstruction}

Animals were imaged using $40 \mathrm{kVp}, 400 \mathrm{~mA}$, and $12.5 \mathrm{msec}$ exposure time. This low-kVp radiographic technique was chosen to enhance soft-tissue contrast within the animal. Each image set consisted of 380 projections regularly spaced over a circular orbit of $190^{\circ}$. Each dataset acquisition per animal lasted for 8-10 minutes and led to an average absorbed dose of 27 cGy. All projections were acquired with ventilatory synchronization at end-expiration. Projection images were 


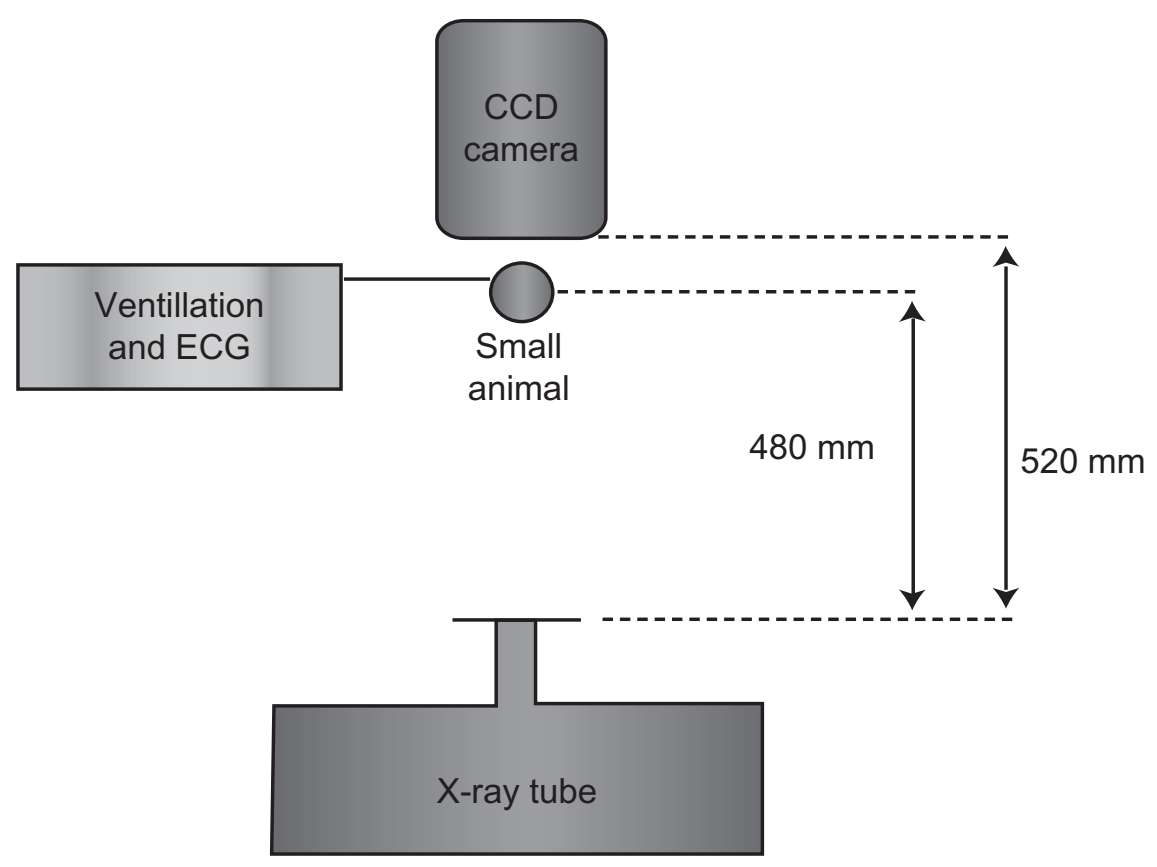

Figure I Placement of the animal in the micro-CT gantry.

Abbreviations: CCD, charge-coupled device; CT, computed tomography; ECG, electrocardiograph.

used to reconstruct tomograms with a Feldkamp algorithm using Parker weighting (Cobra EXXIM, EXXIM Computing Corp., Livermore, CA). The reconstructed datasets were 3D image arrays $5.12 \mathrm{~cm}^{3}$ in size with $(0.1 \mathrm{~mm})^{3}$ voxels.

Contrast enhancement was measured in regions of interest (ROIs) in the tumor as well as in the muscle. To tumor ROI was confined to within a vascular enhancement region within each tumor. The muscle region was approximately $5 \times 5 \mathrm{~mm}$ chosen on the contra-lateral flank of the animal. Tumor volumes and characteristics were obtained by hand segmentation using ImageJ software (http://rsb.info.nih. gov/ij/). Qualitative comparison was made of microvessel distribution, and regions of necrosis between $\mathrm{CT}$ images and corresponding histological sections.

\section{Results}

The liposomal contrast agent produced an average CT number of $861 \pm 180 \mathrm{HU}$ (mean \pm standard deviation) in tumor vasculature and $535 \pm 113 \mathrm{HU}$ in muscle, for an average contrast difference of $326 \pm 151 \mathrm{HU}$. Within the tumor, vessels as small as 200-300 microns were readily visible. The average tumor volume determined by hand segmentation was $0.52 \pm 0.12 \mathrm{~cm}^{3}$. Enhancement, presumably of the microvasculature, of the tumor was clearly apparent on all images of the tumor (Figure 2).

Enhancement persisted for several hours post-injection (Figure 3). Even at four hours post-injection, the tumor signal remained high, especially in comparison to the signal in normal musculature. Figure 4 shows the
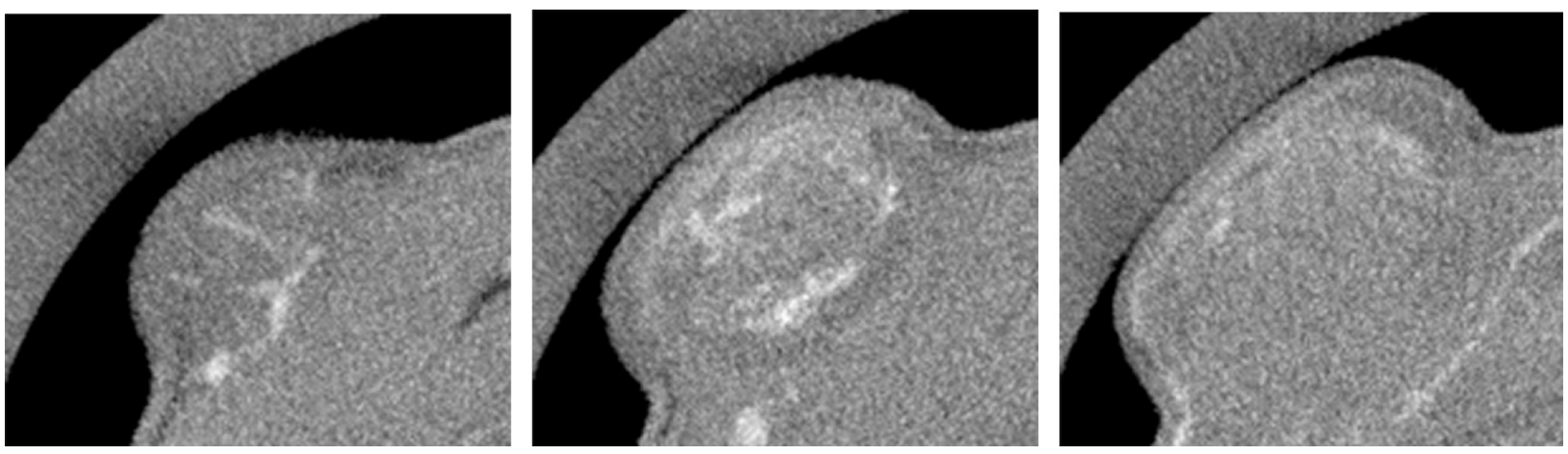

Figure 2 Three tomographic images of an R3230AC tumor in a rat. Enhancement is clearly visible. 


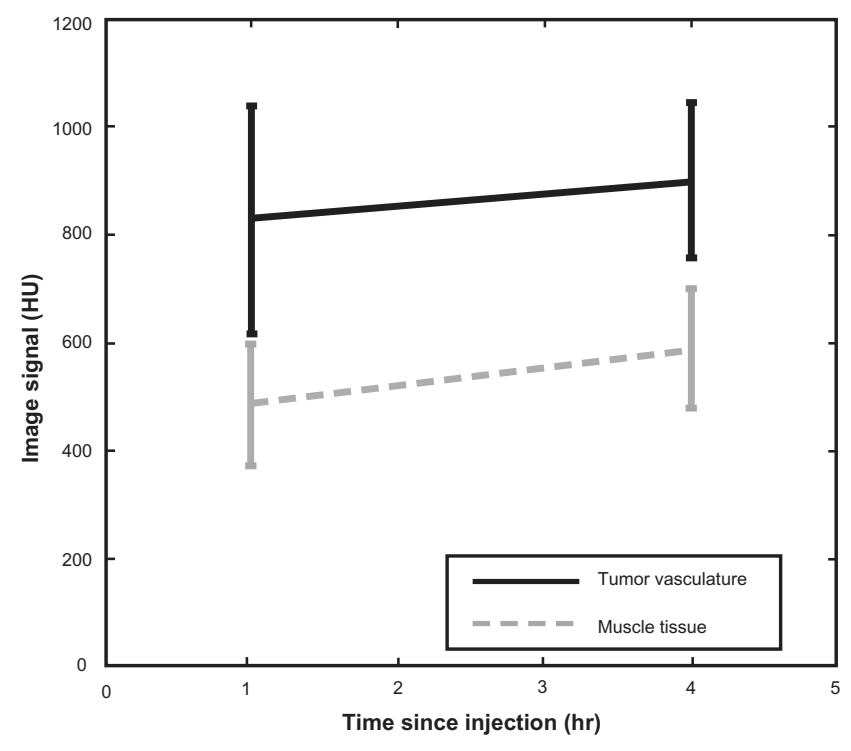

Figure 3 Image value (in $\mathrm{HU}$ ) at two time points for the tumor vasculature and normal tissue. Error bars reflect the standard deviation of the values within the ROI. Abbreviations: $\mathrm{HU}$, Hounsfield units; ROI, region of interest.

tumor enhancement at 1.2 and 4.2 hours after injection. The findings show the continued presence of the nanoparticle contrast agent in the blood pool at four hours post-injection.

\section{Discussion and conclusions}

Comparison between the micro-CT images and corresponding histological sections allows for interpretation of the imaging findings (Figure 5). Blood vessels are present on histological sections in the same areas where contrast enhancement is noted on the CT images. Moreover, evaluation of two typical regions of the tumor, a central slice through the core of the mass, and a peripheral slice along the capsular margin yield additional insight. A region of central necrosis (pink-staining on histological section) is readily evident on the central section and corresponds to region of subtle hypodensity on the corresponding CT image (Figures 5a-c). This finding is in keeping with the known behaviour of liposomes which circulate within the bloodpool without significant extravasation. Therefore, enhancement would only be expected within the viable (purple-staining portions) of the mass. On the peripheral section, blood vessel proliferation is demonstrated along the margins of the tumor on histological section and correspond to peripheral enhancement of the tumor.

The findings demonstrate the utility of the nanoparticle contrast agent for imaging angiogenesis in breast cancer. The agent enabled an attenuation difference between the tumor vasculature and the muscle of $326 \pm 151 \mathrm{HU}$, allowing visualization of the 3D vascular architecture. This level of attenuation difference also enables estimations of tumor volumes and vascular percentages. As vascular enhancement was observed at four hours post-injection, its residence time in the blood pool was at least four hours. This was significantly longer enhancement, on the order of hours, compared to traditional, non-encapsulated contrast agents. In addition, the liposomal envelope shields the body contrast agent, resulting in elimination via the liver and spleen rather than excretion via the kidneys.

Several other liposomal-based agents have been utilized. Liposomes encapsulating both iodine-based and gadolinium-based molecules have been developed for multimodality imaging using CT and MRI. ${ }^{16,17}$ These agents were prepared with a diluted starting iodine solution to incorporate the gadolinium-chelates, thus resulting in low iodine concentration within each liposome. Prior studies have not focused on breast cancer cell lines as a precursor for human applications.
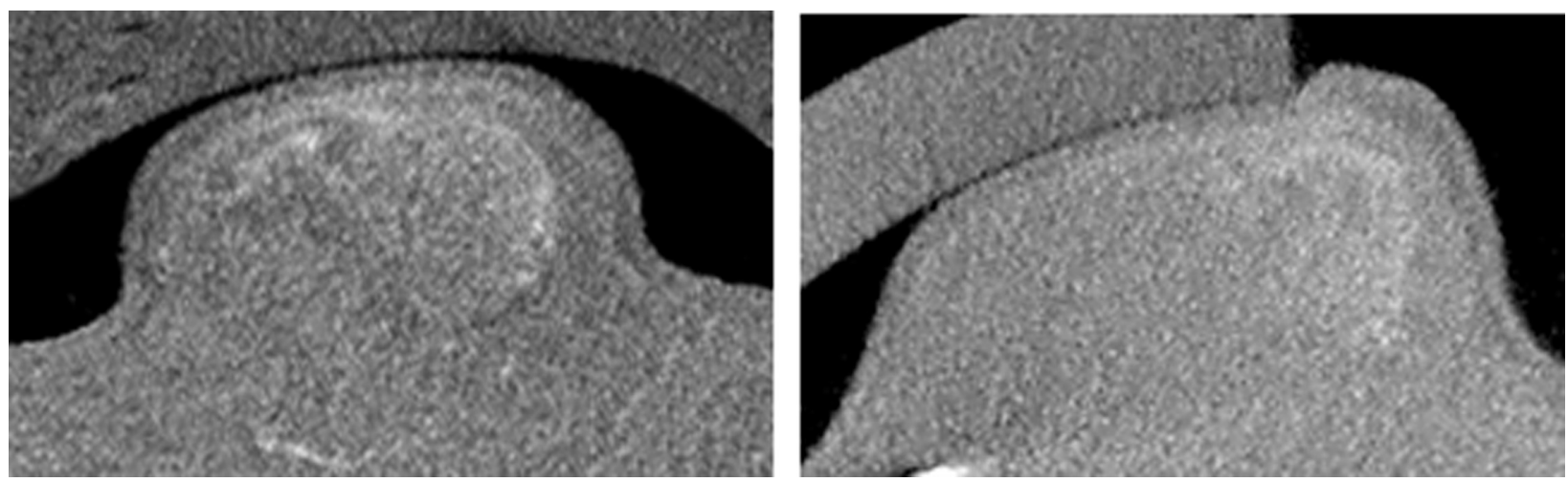

Figure 4 Persistent enhancement of the tumor of a rodent several hours after contrast injection of a rodent with liposomal contrast. These two images were acquired I.2 hours (left) and 4.2 hours (right) after contrast injection. The images reflect positioning differences between two acquisitions. 
Liposomes containing iodine conjugated to the lipids have also been developed. ${ }^{18}$ The conjugation of iodine to lipid results in low iodine:liposome ratio compared to our formulation, wherein the iodinated molecules are encapsulated within the core interior. Although not used in this study, liposomes with an even higher concentration of iodine (eg, $100 \mathrm{mg} / \mathrm{ml}$ ) have been successfully used..$^{10}$ Increases in iodine concentration in combination with a diffusion-optimized size for the liposomes would allow for even better visualization of tumors in small animals and may open the door to delayed micro-CT imaging studies to examine tumor leakage via contrast agent extravasation.

While these findings are qualitative, due to the pilot nature of the study, they suggest that a liposomal, nanoparticle contrast agent is a promising tool for micro-CT evaluation of tumor vascular architecture in small animals. As such, this approach may be useful for the study of tumor angiogenesis including the evaluation of antiangiogenic therapies in small animals with potential extensions and applications to human imaging. The findings pave the way for a future more extensive study with a larger cohort of animals to provide statistically justified quantitative results.

\section{Acknowledgments}

All imaging was performed at the Duke Center for In Vivo Microscopy, an NCRR/NCI National Resource (P41 RR005959/U24 CA092656). The research was supported in part by grants from NIH/NCI (R21 CA124584) and Prevent Cancer Foundation. The authors report no conflicts of interest in this work.
A

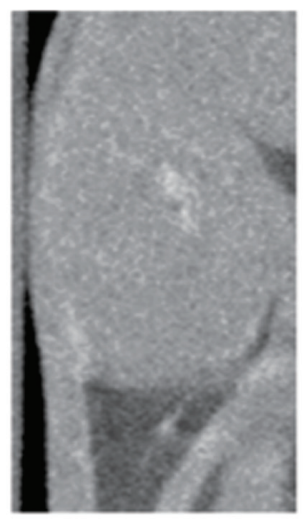

D

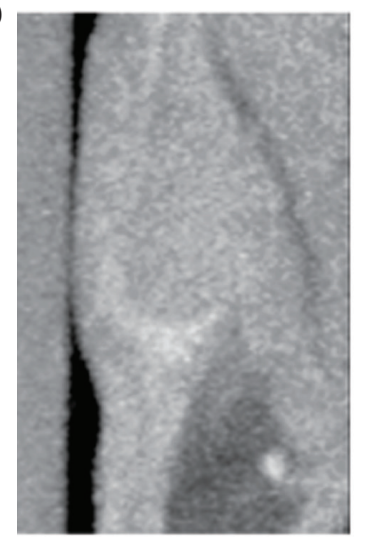

B

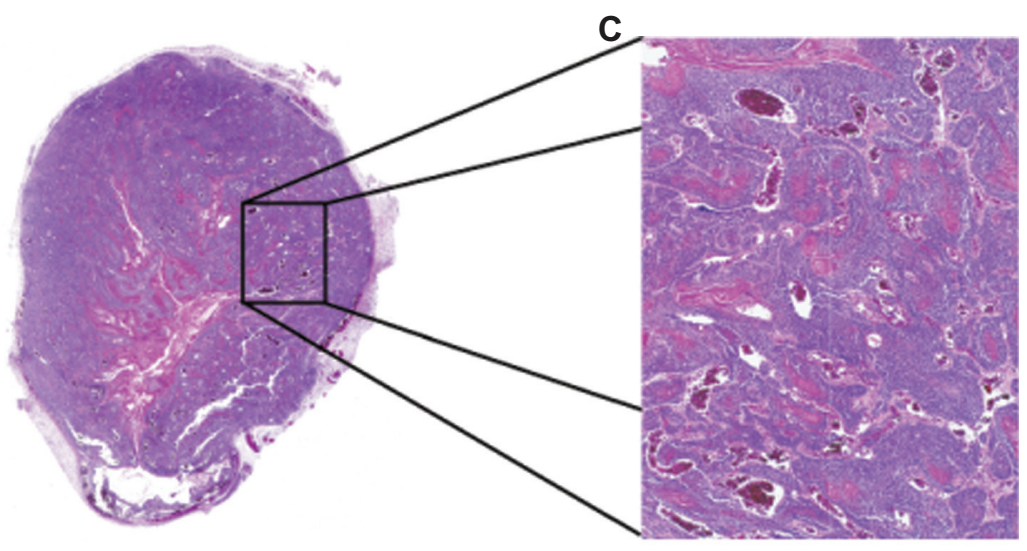

E
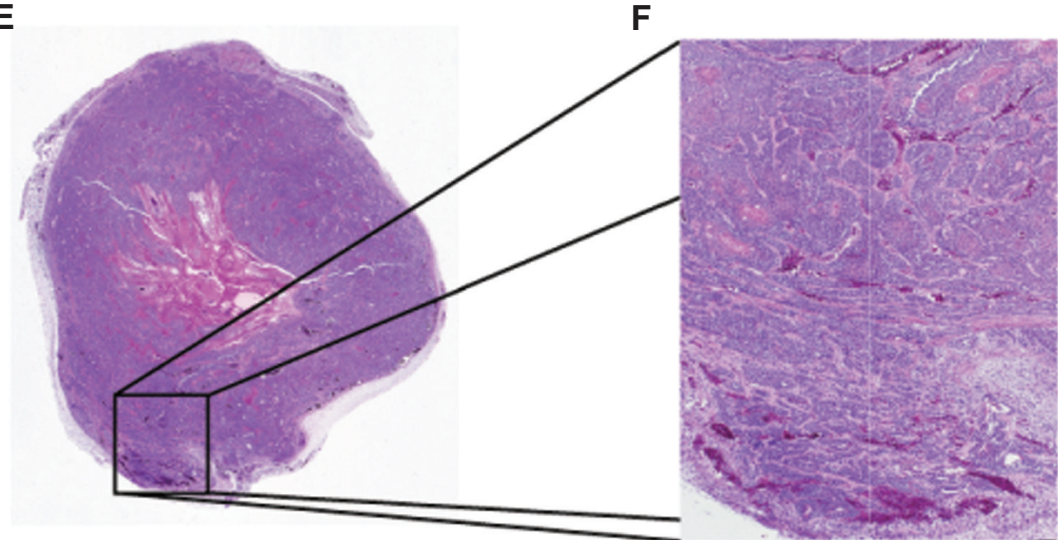

Figure 5 Micro-CT images and micrographs of histological sections of the same R3230AC tumor implanted in a rat. For a central section of the tumor, this figure shows the CT image A) the corresponding histological section micrograph (low-power) B) and a high-power micrograph of the region contained within the box C). Focal enhancement demonstrated on the micro-CT image corresponds to a cluster of blood vessels in corresponding region of the histological section. For a peripheral region of the tumor, this figure shows the CT image $\mathbf{D}$ ) the histological section (low-power micrograph) E) and a high-power micrograph of the region contained within the box $\mathbf{F}$ ). In this slice, enhancement of the rim of the tumor is demonstrated. The corresponding region of the histological section demonstrates significant blood vessel growth. While the $\mathrm{CT}$ and pathology findings cannot be correlated quantitatively, they show similar qualitative indications. Abbreviation: CT, computed tomography. 


\section{References}

1. Schneider BP, Miller KD. Angiogenesis of breast cancer. J Clin Oncol. 2005;23:1782-1790.

2. Ponce AM, Viglianti BL, Yu D, et al. Magnetic resonance imaging of temperature-sensitive liposome release: drug dose painting and antitumor effects. J Natl Cancer Inst. 2007;99:53-63.

3. Hauck ML, LaRue SM, Petros WP, et al. Phase I trial of doxorubicincontaining low temperature sensitive liposomes in spontaneous canine tumors. Clin Cancer Res. 2006;12:4004-4010.

4. Chen Q, Tong S, Dewhirst MW, Yuan F. Targeting tumor microvessels using doxorubicin encapsulated in a novel thermosensitive liposome. Mol Cancer Ther. 2004;3:1311-1317.

5. Kong G, Anyarambhatla G, Petros WP, et al. Efficacy of liposomes and hyperthermia in a human tumor xenograft model: importance of triggered drug release. Cancer Res. 2000;60:6950-6957.

6. Needham D, Anyarambhatla G, Kong G, Dewhirst MW. A new temperature-sensitive liposome for use with mild hyperthermia: characterization and testing in a human tumor xenograft model. Cancer Res. 2000;60:1197-1201.

7. Wu NZ, Braun RD, Gaber MH, et al. Simultaneous measurement of liposome extravasation and content release in tumors. Microcirculation 1997;4:83-101.

8. Ghaghada KB, Bockhorst KH, Mukundan Jr S, Annapragada AV, Narayana PA. High-resolution vascular imaging of the rat spine using liposomal blood pool MR agent. AJNR Am J Neuroradiol. 2007;28:48-53.

9. Zheng J, Perkins G, Kirilova A, Allen C, Jaffray DA. Multimodal contrast agent for combined computed tomography and magnetic resonance imaging applications. Invest Radiol. 2006;41:339-348.
10. Mukundan Jr S, Ghaghada KB, Badea CT, et al. A liposomal nanoscale contrast agent for preclinical CT in mice. Am J Roentgenol. 2006;186:300-307.

11. Montet X, Pastor CM, Vallee JP, et al. Improved visualization of vessels and hepatic tumors by micro-computed tomography (CT) using iodinated liposomes. Invest Radiol. 2007;42:652-658.

12. Braun RD, Lanzen JL, Dewhirst MW. Fourier analysis of fluctuations of oxygen tension and blood flow in R3230Ac tumors and muscle in rats. Am J Physiol Heart Circ Physiol. 1999;277:H551-H568.

13. Hedlund LW, Johnson GA. Mechanical ventilation for imaging the small animal lung. ILAR J. 2002;43:159-174.

14. Badea CT, Hedlund LW, Johnson GA. Micro-CT with respiratory and cardiac gating. Med Phys. 2004;31:3324-3329.

15. Kalender W. Computed Tomography: Fundamentals, System Technology, Image Quality, Applications. Munich, Germany: Publicis MCD Verlag; 2000.

16. Zheng J, Jaffray D, Allen C. Quantitative CT imaging of the spatial and temporal distribution of liposomes in a rabbit tumor model. $\mathrm{Mol}$ Pharm. 2009;6:571-580.

17. Montet X, Pastor CM, Vallée JP, et al. Improved visualization of vessels and hepatic tumors by micro-computed tomography (CT) using iodinated liposome. Invest Radiol. 2007;42:652-658.

18. Elrod DB, Partha R, Danila D, Casscells SW, Conyers JL. An iodinated liposomal computed tomographic contrast agent prepared from a diiodophosphatidylcholine lipid. Nanomedicine. 2009;5:42-45.
International Journal of Nanomedicine

\section{Publish your work in this journal}

The International Journal of Nanomedicine is an international, peerreviewed journal focusing on the application of nanotechnology in diagnostics, therapeutics, and drug delivery systems throughout the biomedical field. This journal is indexed on PubMed Central, MedLine, CAS, SciSearch $\AA$, Current Contents ${ } /$ Clinical Medicine,

\section{Dovepress}

Journal Citation Reports/Science Edition, EMBase, Scopus and the Elsevier Bibliographic databases. The manuscript management system is completely online and includes a very quick and fair peer-review system, which is all easy to use. Visit http://www.dovepress.com/ testimonials.php to read real quotes from published authors. 